\title{
OBSERVATIONS OF COMET a 1902 (BROOKS)
}

This comet was observed here on April 16th (astronomical date), the day on which the telegram announcing its discovery was received. A single setting in each coördinate was obtained through clouds on the following morning (April 17th), but no further observations could be secured, owing to unfavorable weather conditions. After the comet had passed the Sun, it was looked for with the 12 -inch telescope on several nights, and with the 36 -inch on one night.
No trace of it was seen in the positions predicted from the parabolic elements nor in those derived from the short period ellipse computed by Professor Leuschner. At the time of the observation which follows, the comet was estimated as equal in brightness to a star of 8.5 magnitude. A straight tail, extending directly away from the Sun, could be traced fully half a degree. The condensation in the coma was well-defined, but there was no stellar nucleus.

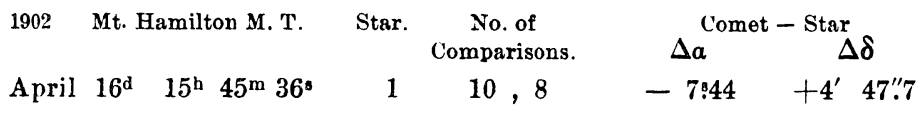

Star.

1

$23^{\text {h }} 21^{\mathrm{m}} 14 ! 61$
Red. to app. place.

$-0.9$
Authority.

A.G. Berlin B. 8975

November 22, 1902.

R. G. Aitken. 